FORMATION Formation emploi

Revue française de sciences sociales

135 | Juillet-Septembre 2016

L'économie verte : rupture ou adaptation de la

formation et de l'emploi ?

\title{
Le secteur de l'installation solaire : encore peu de place pour les entrepreneurs les plus écologiques
}

In the field of solar installing, there is still little room for the most ecological entrepreneurs

Wirtschaftszweig Solaranlagen : immer noch wenig Raum für die umweltbewusstesten Unternehmer

El sector de la instalación solar : todavía poco espacio para los empresarios más ecológicos

Hubert Amarillo

\section{OpenEdition}

Journals

Édition électronique

URL : http://journals.openedition.org/formationemploi/4817

DOI : 10.4000/formationemploi.4817

ISSN : 2107-0946

Éditeur

La Documentation française

Édition imprimée

Date de publication : 12 octobre 2016

Pagination : 115-135

ISSN : 0759-6340

Référence électronique

Hubert Amarillo, « Le secteur de l'installation solaire : encore peu de place pour les entrepreneurs les plus écologiques », Formation emploi [En ligne], 135 | Juillet-Septembre 2016, mis en ligne le 12 octobre 2018, consulté le 21 décembre 2020. URL : http://journals.openedition.org/formationemploi/4817 DOI : https://doi.org/10.4000/formationemploi.4817 


\title{
Le secteur de l'installation solaire : encore peu de place pour les entrepreneurs les plus écologiques
}

Hubert Amarillo

Chargé d'études au Céreq, sociologue du travail

Résumé

\begin{abstract}
Le secteur de l'installation solaire : encore peu de place pour les entrepreneurs les plus écologiques

L'installation solaire photovoltaïque est un nouveau marché sur lequel interviennent trois types d'entrepreneurs : les " solaristes ", caractérisés par leur conviction écologique, les artisans traditionnels et les poseurs sous-traitants. Pour chaque type, la qualification représente une réalité spécifique, socialement encastrée. Les "solaristes ", artisans les plus légitimes pour "justifier" (selon la théorie des conventions) la valeur écologique de leur activité, demeurent peu nombreux, faute de réseaux sociaux suffisants pour les porter.
\end{abstract}

Mots clés : métier de l'extraction-énergie, métier du BTP, métier en émergence, chef d'entreprise, écologie,

Abstract

In the field of solar installing, there is still little room for the most ecological entrepreneurs

The solar photovoltaïc installing is a new market in which three types of contractors intervene: the "solar defenders", characterized by their ecological belief; the traditional craftsmen, and the subcontracting installers. It has been noticed that qualification wasn't an element in which the firms were involved, but it is socially embedded reality. As to the "solar defenders", the most legitímate to "testify" (according convention school) in favor of the ecological order of worth of their activity, they remain few. The reason is the lack of social networks able to support them.

Keywords: occupation in the extraction-energy sector, occupation in the public work industry, emerging occupation, entrepreneur, ecology

Journal of Economic Literature: L 74, Q 01, I 20, M 54

Traduction : Auteur. 
Des figures de l'entrepreneuriat émergent dans le domaine des énergies renouvelables, en particulier l'énergie solaire. Différentes approches de la sociologie économique peuvent être utilement mobilisées pour appréhender les dynamiques entrepreneuriales en cours, dans ce contexte où de nouveaux marchés se constituent.

En premier lieu, la sociologie économique nous invite à dépasser une conception schumpétérienne qui voit dans l'entrepreneur - individuel ou collectif - un acteur isolé, un " héros singulier ", innovateur, qui prend des risques, et à l'affût d'opportunités nouvelles pour faire du profit. Elle le réinscrit dans des appartenances et des relations sociales : l'entrepreneur et l'activité économique qu'il réalise peuvent alors être saisis dans les multiples encastrements sociaux au sein desquels ils se déploient (Zalio, 2009. Plus encore, l'encastrement de l'entrepreneur dans ces réseaux relationnels et la position qu'il y occupe apparaissent comme autant de supports à sa performance économique (Granovetter, 2003). Par ailleurs, l'entrepreneur doit lui-même être considéré comme le fruit d'une construction sociale. Certaines conditions, à la fois sociales, culturelles, organisationnelles ou juridiques, doivent être réunies pour rendre possible son engagement dans l'activité économique. Ainsi, cette catégorie est hétérogène et différenciée socialement, notamment à travers la diversité des carrières et des trajectoires (Chauvin et al. 2014).

En second lieu, pour la sociologie économique, l'activité économique et le marché lui-même sont des constructions sociales. Il ne s'agit donc plus de considérer l'entrepreneur comme un agent aux préoccupations uniquement économiques. L'émergence d'activités et de marchés nouveaux sont des révélateurs privilégiés des processus sociaux à l'œuvre. Neil Fligstein $(1996$; 2005) insiste sur le rôle des États et des gouvernements dans l'émergence et la structuration de nouveaux débouchés économiques. A travers la loi et les règles, les politiques fiscales, ils orientent l'activité entrepreneuriale (définition des conditions de la concurrence et des conduites stratégiques compétitives). Ils offrent ainsi aux nouveaux marchés les structures capables de leur fournir la stabilité et la régularité nécessaires à l'engagement des entreprises. Les entrepreneurs eux-mêmes essaient de participer à ce processus et de l'influencer. Pierre Bourdieu (2000) y voit l'occasion d'une lutte au cours de laquelle les entreprises concernées cherchent à occuper des positions qui leur soient favorables dans les champs économiques ou sectoriels en formation.

L'espace social de l'installation solaire représente ainsi un champ de force ou de lutte (relations et interactions entre acteurs). Les positions des individus y sont déterminées par les différentes formes de capital qu'ils possèdent (Bourdieu, 1980). En détenant les capitaux les plus valorisables dans cet espace, c'est-à-dire en occupant les positions dominantes, certains acteurs peuvent définir les régularités et les règles du jeu dans le sens qui leur est le plus favorable. 


\section{Encadré 1 : Méthodologie}

Cet article mobilise les résultats de deux recherches réalisées entre 2009 et 2013, dans la même région. La première (2009) ${ }^{(1)}$ étudiait les rapports entre qualifications et performance énergétique et environnementale dans le bâtiment, à l'échelle d'un département. Elle s'est notamment intéressée au second œuvre, dont fait partie l'installation solaire.

La seconde a été menée, en 2013, dans le cadre de l'Observatoire Hommes Milieux (CNRS). Ses matériaux d'entretien ont été utilisés, notamment afin d'actualiser nos précédentes données.

Notre échantillon de 25 entreprises a été sélectionné à partir de quelques entretiens exploratoires, et de l'étude des sites internet d'un annuaire départemental comprenant 50 installateurs. Cette phase de l'enquête a fait apparaître les variables pertinentes ${ }^{(2)}$ pour discriminer les entreprises concernées : 1 / la présence d'une activité d'installation solaire dominante ou non au sein de l'activité de l'entreprise ; 2 / la logique d'action plus ou moins reproductive ou innovatrice du chef d'entreprise (artisans traditionnels versus solaristes sensibles à l'environnement ou plus encore écologistes) ; 3 / les objectifs (chiffre d'affaires le plus élevé, qualité du travail...) et dispositions personnelles de ces mêmes chefs, liés à leur trajectoire personnelle et professionnelle.

Ces variables ont permis de dégager les trois principaux types d'entrepreneurs composant le secteur de l'installation solaire, dont l'échantillon se répartit comme suit : six " solaristes », huit sous-traitants, onze artisans « traditionnels ».

La catégorie "solaristes » est la moins représentée, car ces entrepreneurs sont encore peu nombreux par rapport aux autres catégories, en particulier les artisans traditionnels. Les soustraitants nous ont conduits à enquêter auprès de leurs donneurs d'ordres, qualifiés de "sociétés commerciales ». Bien que nous distinguions les poseurs des sociétés commerciales, nous avons choisi ici de nous concentrer sur les poseurs, car ce sont eux qui accomplissent les travaux d'installation.

La méthodologie suivie a été la même dans les différentes enquêtes : entretiens semi-directifs auprès des entrepreneurs et des salariés, des organisations professionnelles (FRB, CAPEB) ${ }^{3}$, des acteurs locaux de la formation et de l'emploi (GRETA) ${ }^{4}$, des représentants des collectivités locales; documentation...

25 entretiens ont été menés avec des dirigeants d'entreprises, dans des établissements en majorité artisanaux (moins de dix salariés). Des questions ont été posées sur l'ancienneté dans l'activité, le parcours de formation et professionnel (du chef d'entreprise comme des salariés), les choix économiques et techniques, les critères de recrutement et les relations interentreprises. Une dizaine d'entretiens ont été aussi conduits auprès de salariés afin de préciser les rapports entre les caractéristiques de la main-d'œuvre employée et celles de l'entreprise.

(1) : Financeur : IRES (Institut de recherches économiques et sociales - CGT (Confédération générale du travail).

(2) : Ces différentes variables s'inspirent de la classification de Hernandez E.-M. (2006), « Les trois dimensions de la décision d'entreprendre », Revue Française de Gestion, n 168/169, pp. 337-357.

(3) : Respectivement Fédératon régionale du bâtiment et Confédératon des artisans et petites entreprises du bâtiment.

(4) : Groupement d'établissements (organisme de formation public). 
L'installation d'équipements en énergie solaire, observée à l'occasion de deux recherches ${ }^{1}$ récentes, correspond bien à ce cas de figure d'un marché en construction. On peut l'appréhender au croisement des carrières et profils des entrepreneurs qui s'y positionnent, des luttes qui s'y déroulent et des alliances qui s'y nouent pour le structurer. Au niveau territorial étudié (voir encadré $\mathbf{1}$ ), trois types d'entrepreneurs se positionnent pour saisir le marché du solaire qui s'ouvre : " solaristes ", artisans traditionnels et poseurs sous-traitants. Le groupe des "solaristes" se situe au centre de notre réflexion ; en effet, ses membres semblent a priori justifier le mieux la grandeur ${ }^{2}$ des énergies renouvelables - comparés aux autres groupes d'entrepreneurs - et sembleraient les mieux placés sur le marché.

Règles et normes professionnelles à travers le travail de labellisation et d'agrément, d'une part, compétences et qualifications à travers l'élaboration des formations, de l'autre, sont l'objet d'oppositions et d'alliances entre les parties prenantes.

\section{Trois types d'entrepreneurs concurrents pour l'accès à un marché émergent}

Les préoccupations environnementales et leur prise en compte dans le secteur de la construction font espérer, dans les années qui viennent, le développement d'un marché dans lequel des entreprises tentent de trouver une place ${ }^{3}$.

Des dispositions législatives et réglementaires ont ouvert de nouvelles opportunités économiques à travers un double processus : un ensemble d'obligations réglementaires et un système $\mathrm{d}$ 'incitations financières. Ce processus trouve en partie son origine dans le "Grenelle de l'environnement ", dispositif public lancé fin 2007, visant à prendre d'importantes décisions en matière de développement durable. Dans le secteur de la construction, le "Grenelle " veut accélérer la rénovation thermique des bâtiments et le développement des énergies renouvelables. L'éco-prêt à taux zéro est une des principales mesures financières incitatives en direction des particuliers, permettant d'emprunter sans intérêts pour réaliser des travaux d'isolation. Par ailleurs, le législateur réclame des professionnels une vision globale des bâtiments et, pour obtenir l'éco-prêt, les particuliers doivent réaliser un « bouquet de travaux ", c'est-à-dire plusieurs types d'amélioration ${ }^{4}$. L'aide de l'État peut être abondée par certaines collectivités locales. C'est le cas par exemple du Conseil régional, et

1. Je tiens ici à remercier Annie Lamanthe, professeure à Aix-Marseille Université (Laboratoire d'Economie et de Sociologie du Travail - CNRS), avec laquelle j'ai travaillé sur cette recherche, entre 2010 et 2013.

2. Au sens de la théorie des conventions (Boltanski et Thévenot, 1991), la "grandeur » est une valeur partagée par l'ensemble des membres d'un espace social (une " cité»). Ainsi, dans une cité écologique (non repérée par les auteurs précédents), le " grand " serait l'acteur qui respecte l'environnement.

3. Dans son rapport de début 2010, le Conseil d'orientation pour l'emploi prévoit que la "croissance verte " permettra la création ou le maintien de 600000 emplois, dont la moitié dans le secteur du bâtiment.

4. Par exemple : isoler les murs + poser un chauffe-eau solaire. 
de la Communauté d'agglomération, objet de notre recherche. Au total, ces aides peuvent atteindre 40 à $50 \%$ du coût d'une installation. Ainsi, l'essor de ce type de marché se fonde sur des politiques organisées.

Sur les terrains étudiés, plusieurs types d'entreprises se positionnent sur ce marché. Dans une activité qui émerge et se structure, les visions et les pratiques de l'activité sont diversifiées et en concurrence. Trois types d'entreprises ont été repérés : les « solaristes ", les « traditionnels " et les " poseurs sous-traitants " (cf. tableau 1). On retrouve ici une typologie proche de celle identifiée dans le secteur du bâtiment par Pillemont et al. (1987, cité par Comet, 2007) qui distinguait : les entrepreneurs traditionnels des domaines classiques, les précaires travaillant essentiellement en sous-traitance, les modernistes et les émergents des créneaux innovants. À chaque type correspond un profil d'entrepreneur : trajectoire et formation, conception de l'activité associée à des visions différenciées des qualifications pertinentes.

Tableau 1. Trois types d'entreprises dans le secteur de l'installation solaire

\begin{tabular}{|l|c|c|c|c|}
\hline \multicolumn{1}{|c|}{$\begin{array}{c}\text { Type } \\
\text { d'entreprises }\end{array}$} & Entrepreneurs & Réseaux & Activité & Salariés \\
\hline Solaristes & $\begin{array}{c}\text { Ingénieur, cadre. } \\
\text { Expérience de la grande } \\
\text { entreprise }\end{array}$ & $\begin{array}{c}\text { Forte indépendance } \\
\text { relationnelle. } \\
\text { Construction de réseau profes- } \\
\text { sionnel propre }\end{array}$ & $\begin{array}{c}\text { Concentrée autour de } \\
\text { l'installation solaire. } \\
\text { Performance technique } \\
\text { Association conception/ } \\
\text { installation }\end{array}$ & $\begin{array}{c}\text { Niveau de formation } \\
\text { supérieur à la moyenne du } \\
\text { second cuvre. } \\
\text { Mobilité professionnelle } \\
\text { Qualité de l'exécution } \\
\text { Autonomie }\end{array}$ \\
\hline Traditionnels & $\begin{array}{c}\text { Ancienneté dans le secteur } \\
\text { chauffage/ } \\
\text { plomberie }\end{array}$ & $\begin{array}{c}\text { Relative indépendance } \\
\text { Appartenance à un réseau } \\
\text { professionnel existant }\end{array}$ & $\begin{array}{c}\text { Polyvalence. } \\
\text { Linstallation solaire est un } \\
\text { complémentà l'activité de } \\
\text { chauffagiste/ } \\
\text { plombier }\end{array}$ & $\begin{array}{c}\text { Niveau de formation: } \\
\text { CAP-BEP }\end{array}$ \\
\hline $\begin{array}{l}\text { Qualité de l'exécution } \\
\text { Poseurs } \\
\text { sous-traitants }\end{array}$ & $\begin{array}{c}\text { Anciens ouvriers récem- } \\
\text { ment installés à leur } \\
\text { compte }\end{array}$ & $\begin{array}{c}\text { Dépendance économique } \\
\text { forte par rapport aux } \\
\text { donneurs d'ordre (sociétés } \\
\text { commerciales). } \\
\text { Peu ou pas de relations avec les } \\
\text { réseaux professionnels }\end{array}$ & $\begin{array}{c}\text { Concentrée sur } \\
\text { l'installation. } \\
\text { technique peu élevé }\end{array}$ & $\begin{array}{c}\text { Salariés peu qualifiés. } \\
\text { Présence de manœuvres }\end{array}$ \\
\hline
\end{tabular}

Source : Amarillo H., Lamanthe A. (2011), p. 13.

\subsection{Les " solaristes ", des entrepreneurs convaincus}

Les « solaristes » regroupent des entreprises nouvelles créées sur le créneau des énergies renouvelables. Pour les entrepreneurs, il s'agit ici de faire du solaire la principale activité de l'entreprise (pas de pompes à chaleur ou de climatiseurs), même s'il leur arrive de proposer des équipements pour d'autres énergies considérées comme plus écologiques, chauffage au bois ou géothermie, par exemple. En outre, les solaristes proposent une solution énergétique et environnementale d'ensemble pour leurs clients (" approche globale »), comme des 
travaux de rénovation thermique à réaliser avant de procéder à la pose d'une installation solaire. Enfin, les solaristes ne se limitent pas à installer des équipements, ils cherchent à travailler à la fois sur la conception et la réalisation du projet.

Il s'agit là de nouveaux profils d'entrepreneurs, issus d'autres secteurs, de formation supérieure et ayant souvent occupé des postes de haut niveau dans de grandes entreprises de l'industrie et des services (ingénieur télécommunication, ingénieur systèmes de production, etc.). Ils s'inscrivent dans des trajectoires de reconversion ou de rupture professionnelle. Ici se combinent projet professionnel et posture militante (écologie, choix du solaire). L'orientation de l'activité est à la fois nourrie par cette posture et par la promotion de leur profil d'ingénieurs, de techniciens et leur positionnement en tant que professionnels du solaire : capacité à adapter les équipements aux besoins des clients, à offrir un service global allant du projet jusqu'à l'installation, à évoluer avec les technologies. Mais ces chefs d'entreprise sont aussi de nouveaux arrivants dans le secteur. Ils doivent acquérir des compétences techniques spécialisées, d'une part sur le solaire lui-même et, d'autre part, sur différents aspects techniques connexes, comme la plomberie, le chauffage, la couverture, l'étanchéité,... Ils acquièrent ces connaissances par des stages chez des fabricants, des bureaux d'études spécialisés, des centres de formation, par le recours à des réseaux familiaux et amicaux, des collaborations avec d'autres entreprise, l'embauche de stagiaires et de salariés possédant ces qualifications.

\subsubsection{Un chef d'entreprise en énergies renouvelables}

Après des études supérieures dans le domaine des télécommunications, il travaille pendant onze ans dans une société de micro-électronique dans laquelle il évolue rapidement. Ne se " retrouvant plus " dans les transformations de l'entreprise (logique financière suite à son intégration dans un groupe nord-américain), il profite d'un plan social pour se reconvertir. Il suit alors une formation supérieure en administration de l'entreprise puis crée sa propre structure en janvier 2006. Il bénéficie du soutien de la plate-forme d'initiative locale, de prêts, d'un accompagnement. Il acquiert les compétences techniques qu'il ne possède pas, selon des modalités différenciées : stage chez un fabricant, recours à la famille (un cousin plombier), stage AFPA 5 pour l'obtention de l'agrément,... L'entreprise est installée dans les locaux de la pépinière d'entreprises de la Communauté d'agglomération :

"On voit arriver de nouveaux profils dans la profession, avec les énergies renouvelables. Des personnes qui viennent à cette activité pour des convictions écologistes, souhaitant se mettre à leur compte, avec la volonté d'être autonomes, tout en étant en accord avec ses idées et en ayant l'impression de faire quelque chose, d'apporter une petite contribution, ce qui fait que l'activité n'est pas purement commerciale.» (Chef d'entreprise, 46 ans, ingénieur de formation)

5. AFPA : Association nationale pour la formation professionnelle des adultes. 
Dans ces entreprises, le niveau de formation des salariés est plus élevé que la moyenne observée dans le second œuvre : beaucoup détiennent un niveau supérieur ou égal au baccalauréat. Selon l'INSEE ${ }^{6}, 55,6$ \% des ouvriers qualifiés actifs du second œuvre du bâtiment possèdent un diplôme de niveau $\mathrm{V}$ (CAP, BEP) - Certificat d'aptitude professionnelle et brevet d'études professionnelles), contre seulement 12,1\% un titre de niveau supérieur. Les salariés sont eux aussi porteurs de valeurs écologiques, ce qui induit certains rapports au travail et à l'emploi. Ils font preuve d'une forte mobilité professionnelle, motivés par la recherche de l'emploi qui satisfera leurs exigences (écologie, indépendance, réflexion). Ils valorisent un travail où ils apprennent ; ils refusent parfois une logique carriériste. Enfin, leur rapport à la formation est particulièrement intéressant à observer. Ils expriment une volonté d'apprendre par eux-mêmes, que nous pouvons analyser comme une manifestation actuelle d'un phénomène connu depuis longtemps, celui de l'autonomie ouvrière ${ }^{7}$. Cette motivation militante peut expliquer que les solaristes déclarent ne pas rencontrer les difficultés de recrutement auxquelles font face la plupart des entreprises du bâtiment.

\subsection{Les « traditionnels » privilégient les métiers du bâtiment}

Nous appelons " traditionnels » les dirigeants d'entreprises dont l'activité principale est située dans un corps de métier du bâtiment et qui tentent de développer une activité supplémentaire dans les énergies renouvelables. Se positionnent sur ce marché nouveau les entreprises du bâtiment, qu'elles soient artisanales ou de grande taille, implantées de longue date ou de création récente. Il s'agit d'intégrer cette problématique dans leur cœur de métier, qui peut être le chauffage, la plomberie, l'électricité, de façon complémentaire,... et dans une continuité avec celui-ci. La problématique environnementale est intégrée dans la logique du métier traditionnel. Ces entreprises et les organisations professionnelles du bâtiment considèrent que les énergies renouvelables doivent s'appuyer sur les métiers existants plutôt que d'en susciter de nouveaux. Cette position est aussi motivée par la prudence ; le marché apparaît en effet à la fois porteur de développement mais il est récent, incertain, et fragile (forte dépendance vis-à-vis des aides publiques) :

"On fait pas de pub, ni d'annonces à la radio, donc, on dit aux gens, ben, voilà, il y a plein de gens qui se sont créés là-dessus [sic], parce que c'est à la mode, et puis c'est un marché porteur. Nous, on a une autre activité à côté qui fait, que, ben, quand le solaire marchera plus, on sera encore là; on dit pas qu'on va faire du solaire ad vitam aeternam, sill n'y a plus de marché, on n'en fera plus. En revanche, si vous faites une installation avec nous, dans 20 ans, nous, on a plus de chances d'être là que le type qui fait que ça,... » (Responsable secteur solaire, entreprise d'électricité)

6. Recensement Général de la Population de 1999, au niveau régional.

7. Cf. la tradition du compagnonnage chez les ouvriers de métier décrite par Piotet (2002). 


\subsubsection{Un artisan récemment installé}

Ce responsable s'est installé comme artisan électricien il y a onze mois. Cette installation fait suite à un plan social dans l'entreprise qui l'employait et aux difficultés rencontrées, à 51 ans, pour retrouver un emploi. Il possède au départ un CAP-BEP en électricité, puis a passé différents diplômes, jusqu'au BTS (brevet de technicien supérieur) électrotechnique. Il a travaillé dans plusieurs grandes entreprises, a été cadre. Il a obtenu les qualifications qui lui ont permis d'obtenir l'agrément photovoltaïque (formation dans un centre émanant des organisations professionnelles du génie électrique et énergétique). Il réalise à la fois des chantiers en électricité et en installations photovoltaïques. Pour lui, le photovoltaïque "ça fait partie de l'électricité ». Il travaille seul. Il voit dans les énergies renouvelables un secteur plus porteur que le seul domaine de l'électricité, et à même de lui apporter travail et revenu.

\subsubsection{Une grande entreprise spécialiste en électricité}

Cette entreprise existe depuis dix ans (reprise d'une structure pré-existante). Elle emploie 140 salariés. Elle répond à des appels d'offres émanant de promoteurs immobiliers. Elle vient de créer un secteur solaire afin de se positionner sur ce créneau. Le fils du dirigeant en a été nommé responsable. Un commercial a été embauché, il travaillait précédemment dans une entreprise spécialisée dans les énergies renouvelables. Quatre salariés ont été formés à l'installation du matériel à l'occasion de formations organisées pour l'obtention de l'agrément ; ils ont suivi des formations en couverture et en charpente. Ils bénéficient aussi d'une aide à la formation de la part des fabricants de matériel. Pour l'instant, ces ouvriers ne sont pas spécialisés ; ils partagent leur travail entre les chantiers d'électricité et ceux d'installation solaire :

"Ben, nous, justement, on va se différencier sur le fait qu'on est une société où le caur de métier c'est l'électricité, donc ça, ça fait partie d'une de nos activités; donc ça, c'est important, contrairement à d'autres sociétés qui ne font que ça; donc, ça veut dire que nous, on a un savoir-faire, qui est vraiment de, on est des électriciens, le photovoltaïque, c'est produire de l'électricité, donc, ça fait vraiment partie de notre métier... " (Commercial)

Ces traditionnels se caractérisent par un niveau de formation " plancher " des salariés CAP/BEP - dans les métiers du bâtiment. Dans le même temps, le développement d'une activité dans le domaine des énergies renouvelables nécessite l'acquisition de nouvelles connaissances et compétences : sur le matériel, notamment, et dans d'autres spécialités du bâtiment (par exemple en couverture et étanchéité pour les électriciens ou les chauffagistes). Celles-ci ne sont cependant pas considérées comme spécifiques ou spécialisées, mais s’intègrent aux métiers de base. 


\subsubsection{Un chef d'entreprise chauffagiste}

Ce chef d'entreprise entre en 1979 dans l'entreprise familiale et succède à son père en 1985. L'activité d'installation solaire débute dès les années 1980 ; elle représente $4 \%$ de l'activité totale. L'entreprise emploie dix-sept permanents et cinq intérimaires. Elle est membre de la CAPEB et d'un regroupement d'entreprises (association de professionnels des énergies renouvelables). Le chef d'entreprise suit actuellement une formation mise en place par la CAPEB pour obtenir le label Eco Artisan :

"Au début [les énergies renouvelables], ceétait quelque chose de nouveau, mais très proche de notre travail habituel. On faisait la même formation car on traitait la même chose, l'eau sanitaire, mais avec seulement le moyen qui changeait (le solaire). Il s'agissait aussi de ballons, mais qui utilisaient d'autres énergies. Aujourd'hui, ça reste très basique, seuls les capteurs et la régulation sont spécifiques au solaire. On a fait des formations seulement pour comprendre le fluide caloporteur (un type d'huile). "

Dans son entreprise, le développement de ce marché s'appuie sur les qualifications et métiers traditionnels : recours à des chauffagistes (pour installer les ballons), plombierszingueurs (pour intervenir sur les toits, réaliser l'étanchéité), frigoristes (pompes à chaleur, climatisation), électriciens. L'acquisition des qualifications est elle-même traditionnelle : apprentissage, embauche d'adultes qui sortent de l'AFPA et formation sur le tas dans l'entreprise. Le solaire nécessite cependant l'acquisition de nouvelles compétences :

"Tous les gens qu'on a, ils l'ont acquis dans l'entreprise, la connaissance du solaire (...), ils sont allés chez des fabricants, ils sont allés faire des stages. "

Selon ce chef d'entreprise, le solaire ne permet pas une spécialisation de l'activité sur ces technologies ; il convient donc de maintenir une offre d'ensemble sur le chauffage. Il insiste donc sur la capacité de son entreprise à traiter la plupart des questions de chauffage, et se prévaut d'une expérience de 20 ans :

"On a appris il y a longtemps, on a fait des stages AFPA de X à la demande de EDF, pour installer les premières pompes à chaleur."

\subsection{Les sociétés commerciales et leurs poseurs sous-traitants font primer la logique financière}

La perspective d'un marché porteur a fait émerger une offre commerciale émanant de sociétés qui ne possèdent pas de compétences techniques dans le domaine. Elles ne souhaitent pas acquérir ces compétences car leur activité est commerciale et parfois, elles peuvent vendre de tout autres produits. Elles ont recours à des installateurs en soustraitance pour poser le matériel qu’elles vendent, installateurs qui sont spécialisés sur une tâche, un produit, un type d'équipement. En fait, ce sont des exécutants : ils réalisent l'installation prévue sur le bon de commande. 


\subsubsection{Une société commerciale}

Cette société compte dix concessionnaires sur le pourtour méditerranéen. Son agence locale emploie une soixantaine de personnes, pour l'essentiel des techniciens-conseil et des télévendeurs. Elle sous-traite la majeure partie des installations des produits qu'elle vend. Ses installateurs n'utilisent que ses produits. Il y a un responsable technique pour sept installateurs. Ce responsable insiste sur l'importance de la possession de l'agrément QUALIPV $^{8}$ pour le choix des installateurs, même si, à ses yeux, elle n'est pas forcément synonyme de compétence professionnelle significative dans le solaire. Son rôle est de contrôler les installations et la qualité, sur la base d'un document rempli par le client.

Ces entreprises installatrices sont en position de subordination. Leurs salariés appliquent les décisions de l'entreprise donneuse d'ordres. Les qualifications attendues sont basiques, liées aux métiers traditionnels du bâtiment. Les entreprises emploient aussi du personnel non qualifié : manœuvres, manutentionnaires. Dans ce créneau, c'est la possession de l'agrément qui prévaut sur tout autre signe de qualité. Elle est nécessaire, même si le principal argument de vente a trait aux avantages financiers et fiscaux.

\subsubsection{Une entreprise installatrice}

Le gérant de cette entreprise est d'origine étrangère. Il s'est installé en 2007. Électricien de formation, il a travaillé toute sa vie dans le bâtiment. Salarié d'une entreprise pendant trois ans, il y a acquis une expérience dans le photovoltaïque. Il s'est lancé dans l'activité car il savait le secteur en croissance. Il a fait de la charpente, et pour lui, c'est un atout, car la pose de panneaux induit des problèmes d'étanchéité :

"On travaille pour d'autres sociétés, qui, elles, vendent soit aux particuliers, soit aux professionnels. Et nous, on agit au niveau de l'installation, seulement. On commercialise pas le produit, on n'est quinstallateur..." (Gérant d'entreprise de pose, électricien, non européen)

L’entreprise compte deux " techniciens poseurs ». Leur compétence est centrée sur la pose. Elle emploie aussi un "manœuvre ", alors que ce profil ne se retrouve pas chez les autres installateurs indépendants rencontrés, les tâches de manutention étant réalisées par les ouvriers installateurs. Pour recruter, le gérant n'exige pas de titre professionnel, l'expérience peut être suffisante. En revanche, il exige les titres nécessaires à certaines catégories d'installation :

«(...) les qualifications quion demande pour travailler dans ce domaine, c'est des agréments, tout simplement. C'est le QUALIPV." (Idem)

8. Cet agrément est un document obligatoire pour réaliser toute installation photovoltaïque. 
Il considère que sa situation est peu avantageuse, d'un double de point de vue : financier, puisque ce sont les sociétés commerciales qui captent la plus grosse partie de la marge ; et en raison de sa position d'intermédiaire entre le commercial et le client, qui nécessite des ajustements entre le matériel vendu et le besoin réel du client :

"Le problème, c'est qu'il y a beaucoup de commerciaux qui vendent des produits, mais on se rend compte, nous, quand on arrive à la maison pour poser ce qu'ils ont acheté, on constate qu'il y a beaucoup de problèmes (...) Il y a beaucoup de choses qui sont pas... honnêtes. Normalement, une installation, elle doit être en plein sud, des fois, on arrive, la maison, le toit, il est en plein nord." (Idem)

\section{Des entreprises en concurrence et en lutte pour définir l'activité}

Ces trois types d'entreprises sont en concurrence pour se positionner sur le marché. Elles le sont aussi pour accéder, dans une activité en émergence, aux instances qui contribuent à structurer le marché, à lui donner un cadre normatif et réglementaire. Il s'agit d'orienter les décisions dans un sens qui leur soit favorable et, par là, de limiter l'accès de l'activité aux concurrents. Cette lutte s'exprime de différentes manières : à travers les critiques mutuelles qu'ils s'adressent, les luttes pour la définition des agréments et des labels, l'insertion dans des réseaux permettant la captation de ressources, et enfin à travers la construction de l'offre de formation.

\subsection{Des critiques croisées}

Les trois types d'entrepreneurs ont des visions divergentes de l'activité et des positionnements. Leurs points de vue et pratiques s'opposent ; les entretiens en témoignent, à l'aune des critiques que les uns et les autres s'adressent et qui sont aussi l'occasion de justifier leurs propres positionnements et pratiques.

Les solaristes sont de nouveaux entrants dans un secteur, le bâtiment, dominé par les artisans traditionnels. Ils s'opposent sur leur conception de l'activité, sur les qualifications et compétences légitimes : activité à part entière et spécifique versus intégration dans un cœur de métier traditionnel. Nouveaux venus dans le domaine, les solaristes importent de nouvelles pratiques qui perturbent les routines des artisans. Se joue aussi entre eux une concurrence sociale : les solaristes ont un niveau d'éducation supérieur ; ingénieurs, ils ont occupé des postes à responsabilité dans de grandes entreprises des secteurs de pointe. 


\subsubsection{Critiques adressées aux solaristes par un artisan traditionnel}

"... C'est que nous, le client, on lui propose de faire du solaire, on lui propose de changer sa chaudière, on lui propose de mettre une pompe à chaleur, on lui propose un éventail de possibilités, et nous ne sommes partisans d'aucune; quand les entreprises ne font que du soleil (...) Ils incitent à faire du soleil, même quand ça ne ressemble à rien".

"Ce quion reproche aux entreprises nouvelles : ils restent entre eux. Ils disent que, eux, ils savent faire du solaire et que nous, nous sommes des bricoleurs, que eux ce sont des pros".

\subsubsection{Critiques adressées aux artisans traditionnels et à leurs organisations par un solariste}

"Les artisans traditionnels n'ont rien fait pour développer ce marché. Ils syy mettent maintenant et avec une approche protectionniste, label, restriction du marché à un petit groupe d'entreprises. Ils ont un gros problème de qualification car ils ne sont pas tous capables d'évoluer vers ces technologies, qui sont plus fines".

Selon les entrepreneurs écologiques, les artisans traditionnels «n'ont rien fait pour développer ce marché ». Il s'agit d'une critique classique des catégories sociales les mieux formées et se définissant comme les plus innovatrices (ingénieurs, cadres du secteur privé), à l'égard des catégories populaires (petits artisans, ouvriers, etc.) présentées comme incapables de remettre en cause leurs dispositions professionnelles. En termes conventionnalistes (Boltanski et Chiapello, 1999), la capacité à s'adapter à une situation nouvelle représente une "grandeur» centrale pour les modernistes, tandis que les acteurs traditionnels et populaires sont jugés passifs, donc "petits » et dépassés.

En revanche, solaristes et traditionnels s'entendent pour dénoncer les pratiques des sociétés commerciales à l'égard de leurs sous-traitants. Elles représentent un danger pour l'activité, elles risquent de décrédibiliser l'ensemble des entreprises aux yeux des clients et de faire péricliter le marché. Cette dénonciation commune repose sur un "principe supérieur commun» (Boltanski et Chiappello, 1999) qui les rapproche9.

\subsubsection{Critique des sociétés commerciales et de leurs poseurs sous-traitants}

"Dans l'activité, il y a maintenant des 'vendeurs d'aspirateurs' qui représentent un danger pour nous. Ils ont les dents longues et ce quils veulent, c'est vendre du matériel et des équipements aux particuliers, peu importe si ce sont ces équipements qui conviennent à leurs besoins" (Chef d'entreprise traditionnel).

Dans la " cité domestique " ${ }^{10}$ (Boltanski et Thévenot, ibid.) qualifier des entreprises de "vendeurs d'aspirateurs", c'est les placer sur une échelle de valeur les désignant comme

9. Selon ces auteurs, ce type de principe (ici, la qualité du travail artisanal) permet de réunir des acteurs issus de mondes sociaux (" cités ») différents.

10. La " cité domestique " est un des six mondes sociaux répertoriés par les auteurs des conventions : ce 
" petits » par rapport au " grand » que représente l'artisan traditionnel. Pour les artisans, est « petit » celui qui n'a pour objectif que la vente pour elle-même, qui plus est la vente de marchandises banales, peu complexes techniquement (des aspirateurs), sur un marché de consommation de masse, alors que l'artisan cherche le travail bien fait, pour un client dont il recherche la satisfaction afin de créer un lien de confiance :

"Quand une entreprise a ratissé la zone marseillaise, qu'ils peuvent plus intervenir dans la zone marseillaise, ils vont attaquer le 04, quand ils peuvent plus faire le 04, ils vont faire le 06, quand ils peuvent plus rien faire, ils vont monter plus haut; c'est-à-dire, c'est des gens qui vendent du solaire parce qu'ils vendent du solaire; même, ils vendent des fenêtres, maintenant; donc, c'est le marché porteur, on vend du solaire... c'est pas la volonté de faire une installation chez un client, que le client soit content, satisfait, et qui vous mène d'autres clients, et qui viennent vous voir pour autre chose, de garder son client...»(Chef d'entreprise traditionnel)

Quand aux poseurs sous-traitants des sociétés commerciales :

"... ils ne savent pas vendre, ils ne savent pas concevoir, ils ne savent rien faire, ils posent des capteurs; donc, ils ne sauront jamais se détacher de lui [du fabricant ou de la société commerciale]...» (Chef d'entreprise traditionnel)

\subsubsection{Critiques des fabricants et de leurs poseurs sous-traitants}

"Ily a aussi sur le marché des sociétés qui recherchent des marchés porteurs. Comme J. Au départ, c'était un fabricant [de panneaux photovoltaïques] et un jour, il a dérapé. Il s'est mis à agréer des gens, des installateurs, avec l'idée de vendre son matériel. Ça a ouvert la porte à des gens qui sont des vendeurs, des commerciaux qui sont des tueurs" (Chef d'entreprise traditionnel).

L’appât du gain généré par ce nouveau marché est critiqué par tous, y compris par des poseurs sous-traitants, au nom de la pérennité de l'activité, du respect du client :

"Il y a beaucoup de sociétés qui respectent pas les clients, hein, ils gagnent, ils veulent gagner de l'argent à tout prix, mais... le problème, c'est que ça va pas durer, tout ça... ${ }^{11}$ "(Poseur soustraitant, électricien)

"Moi, je suis pas, je fais pas partie de la vague de ceux qui se sont intégrés dans le solaire, au moment où y a eu les aides, et où y a eu ce fameux rush, où les gens ont plutôt vu ça comme un appât d'argent, que comme une conviction personnelle. " (Chef d'entreprise traditionnel)

\subsection{Des stratégies de distinction : agréments, labels et réseaux}

La création d'une norme, tels qu'un label, une certification ou un agrément, vise généralement à unifier les choix économiques des entreprises, en les incitant à produire des biens et

monde valorise la tradition, la hiérarchie, la proximité et la durée des relations (économiques et sociales), etc. 11. Effectivement, un moratoire de quatre mois sera décrété par le gouvernement Fillon à la fin de l'année 2010. Par la suite, pour le particulier, le photovoltaïque ne repartira pas vraiment. 
des services qui répondent à un standard. Si l'on suit la théorie des conventions, elle aurait tendance à réduire les "disputes $»^{12}$ (Boltanski et Thévenot, op. cit.) portant sur la qualité de ces biens et services et sur la définition des critères qui permettent de l'établir. Pourtant, selon nos observations de terrain, les normes existantes sont loin de faire taire les querelles autour de ces critères de qualité. Les différents segments de l'installation solaire contestent la validité des normes existantes (voir encadré 2) et, le plus souvent, cherchent à promouvoir d'autres critères. En fait, les normes tendent à cristalliser les luttes entre les différentes catégories d'entreprises autour d'intérêts sociaux, économiques et symboliques. Ici aussi, le processus normatif s'encastre dans la construction sociale de l'activité économique.

Selon les solaristes et les artisans traditionnels, les principales certifications en vigueur dans le secteur solaire - QUALISOL et QUALIPV - octroient une distinction rudimentaire. Pour ces deux segments, ces labels fournissent une information sur un niveau plancher de qualité, et n'évaluent pas les différents niveaux de prestation. De concert, ils reprochent à la labellisation solaire d'être trop facilement attribuée. Par ailleurs, elle renseignerait mal les clients sur la qualité des installateurs. Cette certification ne résout donc pas la question de l'information des consommateurs, comme l'évoque ce solariste :

"QUALISOL, c'est un coup de tampon obligatoire, qui n'a aucune valeur. Pour moi. Qui, au contraire, est même gênant pour le client final, c'est-à-dire, on lui vend une étiquette..." Plus encore, il est parfois reproché une opacité dans l'évaluation des installations par les organismes certificateurs.

Dans ces propos cités, nous retrouvons une critique typique des entrepreneurs privés se définissant comme les plus dynamiques. Ils définissent les acteurs détenant un pouvoir, une autorité publique, comme une contrainte, voire une atteinte à la liberté d'entreprendre. Pour ces innovateurs, les autorités existantes sont définies en termes de frein, d'abus de position dominante, d'incompétence face aux changements. Alors qu'ils sont encastrés dans un environnement social et institutionnel particulier, les solaristes soutiennent un discours économique libéral, où ils se représentent eux-mêmes comme un acteur agissant dans un environnement délivré de toute intervention, notamment publique.

12. Pour cette théorie, nous sommes en présence d'une « dispute » lorsque s'affrontent des principes de valeur (grandeurs) différents. 


\section{Encadré 2 : Les normes de qualité pour l'énergie solaire}

QUALIT'EnR : Association fondée début 2006, elle intervient pour promouvoir la qualité des prestations des installateurs des systèmes à énergie renouvelable. Elle gère notamment deux dispositifs de qualité suivants : QUALI SOL (Norme de qualité solaire : solaire thermique) , QUALI PV (Norme de qualité photovoltaïque) $\left(^{*}\right)$. Ses fondateurs sont les cinq entités professionnelles nationales suivantes:

CAPEB : Confédération de l'artisanat et des petites entreprises du bâtiment ;

ENERPLAN : Association professionnelle de l'énergie solaire ;

UCF - FFB : Union climatique de France (Fédération fançaise du bâtiment) ;

UNCP - FFB : Union nationale de couverture plomberie (Fédération française du bâtiment) ;

SER : Syndicat des énergies renouvelables .

$\left.{ }^{*}\right)$ : Ces deux dispositifs donnent accès, après formation, à des agréments obligatoires pour poser des installations solaires thermiques (QUALI SOL) ou photovoltaïques (QUALI PV).

Les solaristes et les artisans traditionnels cherchent de nouvelles distinctions sur le marché. Ils participent à des initiatives visant à construire de nouveaux labels et à les faire reconnaître. Les réseaux dans lesquels se déploient ces initiatives sont différents. Citons le cas d'un solariste qui est membre d'un dispositif concernant le bâtiment durable, initié par le Conseil régional, et dont une des actions prioritaires est la création d'un label destiné au secteur de la construction. Ce dirigeant cherche ainsi à participer à la définition de ce label afin de pouvoir l'orienter favorablement par rapport à sa représentation de l'activité. Ce label donnera une visibilité à la façon dont il conçoit son activité et en sanctionnera une forme de reconnaissance sur le marché. Plusieurs solaristes participent également à une initiative du Conseil régional en faveur des activités innovantes, démarche facilitée par leur spécialisation dans les énergies renouvelables. Le gérant d'une entreprise artisanale, quant à lui, suit la formation initiée par l'organisation professionnelle dont il est adhérent, la CAPEB, en vue de l'obtention du label Eco Artisan qu'elle a créé et qu'elle réserve aux entreprises artisanales.

En revanche, pour les installateurs d'abord motivés par une stratégie commerciale, les agréments existants représentent une certification suffisante, qu'ils mettent en avant auprès de leur clientèle. Pour eux, il s'agit souvent d'un niveau plafond de qualité au-delà duquel l'entreprise ne compte pas aller. Ce niveau est d'ailleurs souvent vécu comme une contrainte. Certaines stratégies de distinction peuvent cependant apparaître aussi dans ce segment. En témoigne la stratégie du dirigeant d'une petite entreprise, au sein d'un réseau de franchisés, qui trouve que l'agrément QUALIPV n'est pas suffisant pour lui permettre de mieux positionner son offre spécifique. À la différence d'autres franchiseurs, ce petit réseau propose une " offre globale " comprenant des appareils de chauffage et des produits de rénovation thermique (isolation des combles, double vitrage, etc.). Ce dirigeant souhaite recruter deux techniciens en installation solaire pour accroître l'expertise de son 
entreprise et former les poseurs sous-traitants afin d'améliorer leur qualification. Cette démarche devrait être validée par un label spécifique à ce réseau.

Ces stratégies de distinction sur le marché s'appuient sur la mobilisation de ressources et l'activation de réseaux et de relations différenciés.

Les solaristes détiennent un capital à la fois culturel et social qui leur permet, à la différence des artisans traditionnels, de nouer plus facilement des relations avec des acteurs publics auprès desquels ils captent les ressources qui leur manquent (financières notamment). Ils refusent d'adhérer aux organisations professionnelles du bâtiment. Ils s'inscrivent plus volontiers dans un réseau de pairs (entrepreneurs partageant un même type de parcours et une même vision de l'activité), au sein duquel ils mutualisent des ressources (locaux, chantiers, compétences, informations,...).

Les entreprises traditionnelles entretiennent une plus grande distance avec les acteurs publics. Elles s'insèrent dans le réseau puissant des organisations professionnelles, qui leur assure l'accès à des formations conduisant à des labels spécifiques. Ces organisations professionnelles tentent ainsi de mettre des barrières à l'entrée de nouveaux prétendants, dont les solaristes font partie. Par exemple, parce qu'il ne possède pas de CAP dans la spécialité, un entrepreneur solariste, ingénieur de formation, ne peut pas s'inscrire au registre des métiers qui lui permettrait d'être reconnu en tant qu'artisan et d'accéder ainsi à certaines labellisations :

"Au début, quand je voulais créer mon entreprise, je suis allé à la Chambre des Métiers. Et là, très simple, jai fait mon mémoire de diplôme d'ingénieur sur la production des capteurs solaires... Et là, on me dit : "non, vous nềtes pas qualifiè.. Il faut qu’ils se remettent à jour, quoi!»

Ces propos illustrent à nouveau notre précédente remarque sur les entreprises innovatrices qui jugent les pouvoirs publics dépassés. On observe enfin la multiplication des réseaux de fabricants de matériel et de sociétés commerciales (franchises, concessions, labellisations privées,...) dans lesquels s'insèrent les poseurs sous-traitants, les entrepreneurs les moins dotés en ressources. Ces réseaux offrent à la fois une possibilité de distinction sur le marché, l'accès à certaines ressources auxquelles seuls ils n'auraient pas accès (accès au marché luimême car ils ne disposent pas des capacités commerciales suffisantes) mais dont, en même temps, ils sont particulièrement dépendants et captifs.

\subsection{Une lutte pour la construction de l'offre de formation}

Depuis deux ans au moment de l'enquête (2010), une formation d' "installateur mainteneur en systèmes solaires et photovoltaïques » est dispensée par un GRETA (Groupement d'établissements) de la Communauté d'agglomération. Cette formation fait suite au constat dressé par des acteurs locaux (Élus, Mission locale, professionnels, GRETA,...) d'un manque en matière de formation et de l'essor des besoins en main-d'œuvre pour faire 
face au développement de l'activité solaire. Cette problématique est peu, voire pas prise en compte dans les appareils de formation ; des tentatives se font jour pour l'intégrer dans les cursus existants, qu'il s'agisse de la formation initiale à l'Éducation nationale ou de la formation pour adultes dispensée par l'AFPA qui revoit ses titres professionnels et prépare ses formateurs.

La mise en place de cette formation révèle les tensions et oppositions entre les conceptions de l'activité et des qualifications. La concertation locale a mis en exergue des points de vue divergents selon les types d'entreprises.

Les divergences ont porté sur le contenu de la formation. Il y a eu des discussions entre professionnels sur les objectifs et finalités à lui donner. Elles ont opposé un important fabricant local et des chefs d'entreprises solaristes et traditionnels. Le premier défendait l'idée d'une formation basique à la pose de panneaux et de chauffe-eaux solaires ; les deux autres, une vision plus large, celle d'une formation de spécialistes capables de réaliser une installation complète :

"Nous, on s'est battu, lors des débats, pour que ces gens là aient une connaissance, derrière le capteur, c'est-à-dire, quand ont met un capteur, on met une régulation et on branche sur quelque chose, savoir sur quoi on branche, savoir ce que c'est un réseau d'eau, parce qu'on prend des gens, qui viennent du chômage, qui sont à des années lumière... donc, on a voulu leur donner la connaissance de la plomberie. Après, on a dit, ça serait bien qu'ils connaissent un peu d'électricité, parce que les régulations, il faut les brancher, donc, on leur a donné une petite formation électrique. Et les formateurs sont allés jusqu'à leur expliquer ce que c'était une chaudière, parce que le solaire se branche sur des chaudières, pour que le gars, quand il va rencontrer une installation dans une entreprise, il va pas arriver : 'je sais poser que des capteurs'... "(Chef d'entreprise traditionnelle)

Des désaccords ont ensuite opposé les solaristes aux traditionnels, sur le profil des personnes qui seraient admises à suivre cette formation (adressée, au départ, à des demandeurs d'emploi). Les solaristes souhaitaient que le recrutement soit le plus ouvert possible, occasion pour eux d'attirer de nouveaux profils dans le secteur :

"Les énergies renouvelables sont une opportunité fabuleuse pour attirer de nouvelles personnes dans le bâtiment, qui ont un niveau élevé de motivation pour les énergies renouvelables (...) Je n'adhère pas à tous les discours des organisations professionnelles, il faut s'ouvrir à des gens venant de l'extérieur, pour faire monter le niveau. " (Chef d'entreprise solariste)

A l'inverse, les traditionnels souhaitaient réserver l'accès à cette formation à des personnes possédant déjà une expérience de chauffagiste. Pour eux, une trop grande spécialisation en solaire ne permettrait pas aux personnes formées de trouver un emploi ou de s'installer dans des conditions optimales de réussite, et ils privilégiaient la polyvalence.

La formation a été ouverte en 2006, et accueille sa deuxième promotion au moment de l'enquête. Cette formation s'adresse à une population de demandeurs d'emploi possédant 
une expérience dans le bâtiment et souhaitant se reconvertir dans l'installation d'équipements solaires ou créer une nouvelle activité. C'est la deuxième formation de ce type en France. La première année, il s'est agi d'une formation de niveau V (de type CAP, BEP), non diplômante, ouverte à des demandeurs d'emploi jeunes et adultes, financée par le Conseil régional : quinze personnes y ont participé. Pour la seconde promotion, l'ambition est plus élevée, notamment à la demande du Conseil régional qui l’a intégrée dans son Plan régional de formation. Il s'agit désormais d'une formation de niveau IV (de type bac ou brevet professionnel), diplômante. La question de la certification a posé problème : il n'existe en effet pas de diplôme de l'Éducation nationale dans le domaine. Cette formation est donc validée par un CQP (Certificat de qualification professionnelle) mis en place par l'UCF (Union climatique de France), organisation professionnelle de la branche, qui a d'abord été réticente à labelliser cette formation. Mais la possibilité que celle-ci débouche sur la certification délivrée par l'UCF a entraîné une refonte partielle de la formation. Le photovoltaïque, non prévu au départ, a dû être intégré et la durée de formation augmentée (1 200 heures, dont 686 en centre). La formation prépare en même temps les stagiaires au $\mathrm{CAP}$ «thermique » afin de conforter leur positionnement dans le domaine du chauffage. Cette offre révèle ainsi les compromis réalisés entre les parties prenantes.

Au final, la CAPEB s'est peu investie, tant financièrement que sur le plan institutionnel, dans cette initiative de formation qui concurrence directement son dispositif " Eco artisan ». L'Union climatique de France, également acteur professionnel centré sur des techniques traditionnelles, n'a consenti que tardivement à valider un CQP. Les apports les plus importants ont émané des acteurs publics : le GRETA a assuré des « investissements lourds en achat de matériel» (Conseiller en formation), et la mission locale a mis en place la formation avec ses propres moyens la première année. Sans compter l'appui du Conseil régional et de la Communauté d'agglomération (commande publique, soutien financier aux particuliers). Matériellement, les solaristes n'ont pu agir qu'en accueillant quelques stagiaires de la formation, "par conviction " (Chef d'entreprise solariste).

\section{Conclusion}

Nos observations révèlent la diversité sociale des entrepreneurs qui se situent sur le créneau de l'installation solaire dans un même espace géographique.

L'entrepreneuriat s'ancre ici dans des mondes sociaux différents : celui des ingénieurs issus des grandes entreprises de technologies de pointe, celui des artisans expérimentés dans le chauffage et la climatisation, celui des ouvriers qualifiés en reconversion,... Cette diversité rejaillit sur les conceptions de l'activité et les positionnements dans l'activité et sur la façon dont ces entrepreneurs justifient leur engagement dans cette dernière (Boltanski et Thévenot, op. cit.). La recherche du profit n'est qu'une motivation parmi d'autres à l'entrée dans l'activité économique. Pour certains, elle correspond à un engagement militant ; pour d'autres, il s'agit de stabiliser un revenu et de se donner les moyens de poursuivre une activité?... Et les réseaux sur lesquels ils s'appuient - marché du travail, fournisseurs et 
clientèles, autres entreprises coopérantes, acteurs publics - se déclinent de manière différente selon le monde social de chaque catégorie d'entrepreneurs.

Les énergies renouvelables constituent un marché en construction. Les entreprises observées sont en concurrence dans le processus qui vise à structurer et stabiliser l'activité et à l'orienter dans le sens correspondant le mieux à leur propre positionnement. Par leurs trajectoires et carrières, les " solaristes ", les " artisans traditionnels » et les "poseurs sous-traitants" sont inégalement dotés en types de capital permettant d'influer sur ce processus (Bourdieu, op. cit.). Ils s'appuient sur des réseaux différents : réseau des acteurs publics locaux et des pairs pour les solaristes ; des organisations professionnelles pour les artisans traditionnels ; réseaux d'installateurs des fabricants et des sociétés commerciales pour les poseurs.

Dans cette lutte, la participation à la définition des labels et agréments représente un moyen, pour chacun, d'accéder à la définition des atouts qui font valeur dans le champ, et de se protéger en posant des barrières à l'entrée et des limites à l'exercice de l'activité pour les concurrents. L'offre de formation, l'accès aux qualifications et leur définition apparaissent eux-mêmes comme des enjeux importants dans cette construction ; ce sont aussi des objets de lutte entre les parties prenantes.

Ces résultats permettent aussi d'éclairer la construction sociale de la qualification de l'entrepreneur. La sociologie économique a évoqué l'encastrement du marché du travail dans les réseaux sociaux (Granovetter, 1974), mais elle a moins exploré la question de l'encastrement de la qualification - c'est-à-dire du phénomène de production et de reproduction des qualités de la force de travail - dans des liens sociaux. Si certains auteurs en sociologie du travail (Naville, 1963 ; Reynaud, 1989) ont bien montré le caractère socialement construit, donc relatif et historique, de la qualification, ils ne l'ont pas définie de manière assez précise et opératoire.

Or, en partant d'une conception relationnelle des acteurs, la sociologie économique apporte une contribution intéressante à la définition de la qualification. Elle révèle que la qualification n'est pas un bien "déjà là ", dans lequel les entreprises "investissent " en fonction d'un calcul de rentabilité à venir (Becker, 1964). Les "investissements " sont souvent liés à des dispositions existantes, non délibérément choisies, qui déterminent des choix d'emblée à la fois sociaux et économiques. Dans ces choix, et notamment dans ceux des relations de partenaires économiques, le calcul de rentabilité pur fonctionne peu. Chacune des trois catégories d'entrepreneurs entretient des liens avant tout parce qu'ils se reconnaissent sur la base de valeurs communes : engagement écologique, savoir-faire traditionnel, adaptation à la demande.

Les " solaristes " échangent entre eux car ils sont d'accord sur un certain nombre de "grandeurs communes", et c'est cet accord qui supporte leur échange (Boltanski et Thévenot, op. cit.). Ces mêmes " solaristes" reflètent le mieux l'idéologie de l'entrepreneur "connexionniste" (Boltanski et Chiapello, op. cit.) de la cité par projets, à travers 
leur refus de la hiérarchie (pas de sous-traitance, refus d'entrer dans les organisations professionnelles traditionnelles et structurées) ou l'accent mis sur la capacité à travailler avec une multiplicité d'acteurs. Par ailleurs, ce sont les parcours professionnels dans la grande entreprise qui apportent les " ressources » utiles pour créer les compétences managériales utilisées sur la "scène sociale » du solaire écologique (Zalio, 2007). De ce point de vue, nous insistons sur le rôle des phénomènes de "découplage " (Grossetti et Bès, 2001), c'est-à-dire de création de nouveaux liens à partir d'un socle social initial dont $\mathrm{ils}^{13}$ se libèrent, pour générer de nouvelles sphères sociales de l'entrepreneuriat. Or, les moments et lieux d'apparition de ces découplages nous sont apparus peu nombreux ; ce qui réduit sans doute les chances de diversification de ces sphères dans les énergies renouvelables, en France. Ainsi, entre 2009 et 2013, le segment des "solaristes " s'est peu développé sur le territoire observé, alors que ces entrepreneurs semblaient les mieux placés pour "justifier" (Boltanski et Thévenot, op. cit.) l'essor du marché de l'énergie solaire photovoltaïque grâce à leur doxa écologique.

Le rôle des pouvoirs publics est finalement central (Bourdieu, op. cit.; Fligstein, 2005). L'État est un acteur principal de l'émergence et de la construction du secteur de l'énergie solaire : par la réglementation, il établit une obligation; par les incitations fiscales, il stimule et solvabilise une demande. Ceci montre l'utilité de l'encastrement de type "structural " ${ }^{14}$ (Steiner, 2007) pour atteindre des objectifs précis. Au niveau territorial étudié, les collectivités locales ont aussi des capacités non négligeables d'intervention dans la construction de ce champ et dans sa structuration : mise en place de dispositifs de soutien aux entreprises, financement de la formation,... Pourtant, la majorité des entrepreneurs interrogés jugent le système d'agréments actuel insuffisant, et recherchent une plus forte régulation de la qualité des produits. Cela indique, si nécessaire, que les réseaux sociaux offrent une structure incontournable, mais insuffisante, pour l'ancrage d'un nouveau marché.

\section{Bibliographie}

Amarillo H., Lamanthe A. (2011), "The social construction of economic activities: entrepreneurs, skills and accreditation in the solar energy sector", paper presented at the 27 EGOS Colloquium, Gothenburg, Sweden, 7-9 July.

Boltanski L., Thénevot L. (1991), De la justification. Les économies de la grandeur, Paris, Gallimard.

Boltanski L., Chiapello E. (1999), Le nouvel esprit du capitalisme, Paris, Gallimard.

13. Il peut s'agir d'un phénomène d'essaimage, où un salarié décide de quitter la grande entreprise qui l'emploie (socle initial) pour créer sa propre entreprise et un réseau d'entrepreneurs écologiques (nouveau socle). 14. Pour la sociologie économique, cette forme d'encastrement repose d'abord sur les réseaux de relations, notamment les réseaux les plus construits. L'exemple type étant celui du réseau d'électricité. 
Bourdieu P. (1980), "Le capital social : notes provisoires ", Actes de la recherche en sciences sociales, 3, pp. 2-3.

Bourdieu P., (2000), Les structures sociales de l'économie, Paris, Seuil, coll. « Liber ».

Chauvin P.-M., Grossetti M. \& Zalio P.-P. (2014) (sous la dir.), Dictionnaire sociologique de l'entrepreneuriat, Paris, Presses de Sciences Po.

Comet C. (2007), « Capital social et profits des artisans du bâtiment : le poids des incertitudes sociotechniques ", Revue française de sociologie, vol. 48, n 1, pp. 67-91

Conseil d'orientation pour l'emploi (2010), Croissance verte et emploi, rapport, Paris.

Fligstein N. (2005), "States, Markets and Economic Growth", in Nee V., Swedberg R. (ed), The Economic Sociology of Capitalism, Princeton University Press, pp. 120-143

Fligstein N. (1996), "Markets as Politics: A Political-Cultural Approach to Market Institutions", American Sociological Review, vol. 61, n 4, pp .656-673

Granovetter M. (1974), Getting A Job. A Study of Contacts and Careers, Harvard University Press, Cambridge, Massachusetts.

Granovetter M. (2003), « La sociologie économique des entreprises et des entrepreneurs », Terrains et Travaux, vol. 1, n 4, pp. 167-206

Grossetti M., Bès M.-P. (2001), « Encastrements et découplages dans les relations scienceindustrie ", Revue Française de Sociologie, vol. 42, n 2, pp. 327-355.

Hernandez E.-M. (2006), "Les trois dimensions de la décision d'entreprendre ", Revue Française de Gestion, n 168/169, pp. 337-357.

Naville P. (1963), "Réflexions à propos de la division du travail ", Cahiers d'étude des sociétés industrielles et de l'automation, $\mathrm{n}^{\circ}$ 5, pp. 232-244.

Piotet F. (2002), La révolution des métiers, Paris, PUF, coll. « Le lien social ».

Reynaud J.-D. (1989), Les règles du jeu, Paris, Colin.

Steiner P. (2007), La sociologie économique, Paris, La Découverte, coll. " Repères ».

Zalio P.-P. (2007), " Les entrepreneurs enquêtés par les récits de carrières : de l'étude des mondes patronaux à celle de la grammaire de l'activité entrepreneuriale ", Sociétés contemporaines, $\mathrm{n}^{\circ} 68$, pp. 59-82.

Zalio P.-P. (2009), "Sociologie économique des entrepreneurs », in Steiner P., Vatin F. (dir.), Traité de sociologie économique, Paris, PUF, coll. «Quadrige Manuel », pp. 573-607. 